\title{
Combate ao Tabagismo no Brasil: a importância estratégica das ações governamentais
}

\author{
Combating smoking in Brazil: \\ the strategic importance of government actions
}

Sandra Tavares da Silva ${ }^{1}$

Mariana Campos Martins ${ }^{1}$

Franciane Rocha de Faria ${ }^{1}$

Rosângela Minardi Mitre Cotta ${ }^{1}$

${ }^{1}$ Departamento de Nutrição e Saúde, Universidade Federal de Viçosa. Av. P H Rolfs S/N, Centro. 36.571-000 Viçosa MG Brasil. sandratavs@yahoo.com.br
Abstract Brazil is a world benchmark in combating smoking and promoting actions through the National Program for Tobacco Control and other Risk Factors for Cancer (PNCTOFR). This literature review was conducted in order to outline the actions of PNCTOFR and identify evidence of their effectiveness in reducing the prevalence of smoking in Brazil. The documents available in the electronic portals of INCA, the National Policy on Health Promotion, the National Tobacco Control Observatory, the World Health Organization and the Pan American Health Organization were used, in addition to the Lilacs, PubMed Central, SciELO and ScienceDirect databases. The reference bases for analysis of the actions were the areas/dimensions proposed by the International Union for Health Promotion and Education. The restriction of the availability, control of marketing and commercialization, educational activities in schools, assistance in primary care for health professionals and the general population, control of consumption in public places and work are some of the actions that contributed to reduction in the prevalence of smoking in Brazil from $32.7 \%$ in 1997 to $14.8 \%$ in 2011. The results indicate the effectiveness of the actions of PNCTOFR, albeit smoking is still a public health problem and needs to be curbed.

Key words Government programs, Tobacco, Health promotion, Evidence
Resumo O Brasil é referência mundial no combate ao tabagismo e desenvolve ações por meio do Programa Nacional de Controle do Tabagismo e outros Fatores de Risco de Câncer (PNCTOFR). Com objetivo de apresentar as ações do PNCTOFR identificando as evidências de sua eficácia na redução da prevalência do tabagismo no Brasil, foi realizado este estudo bibliográfico. Utilizou-se documentos disponibilizados nos portais eletrônicos do Inca, da Política Nacional de Promoção da Saúde, do Observatório da Política Nacional de Controle do Tabaco e das Organizações Mundial da Saúde e Pan-Americana de Saúde, além das bases de dados Lilacs, PubMed Central,SciELO, ScienceDirect. O referencial para análise das ações foram as áreas/dimensões propostas pela International Union for Health Promotion and Education. A restrição da disponibilidade, controle do marketing e comercialização, atividades educativas nas escolas, atendimento na atenção primária à saúde a profissionais da área e à população em geral, controle do consumo em locais públicos e de trabatho são algumas das ações que contribuiram para redução na prevalência de tabagismo no Brasil, de 32,7\% em 1997 para 14,8\% em 2011. Os resultados apontam para a eficácia das ações do PNCTOFR, mas o tabagismo ainda é problema de saúde pública e precisa ser desnormatizado.

Palavras-chave Programas de governo, Tabaco, Promoção da saúde, Evidências 
Introdução

O tabagismo é a causa prevenível mais importante de aproximadamente metade das doenças dos países em desenvolvimento e ameaça minar o seu desenvolvimento econômico e social ${ }^{1-4}$. No Brasil, o tabagismo é considerado problema de saúde pública e seu controle sistemático tem sido realizado desde 1989, quando o Ministério da Saúde (MS), por meio do Instituto Nacional de Câncer (Inca), criou o Programa Nacional de Controle do Tabagismo (PNCT), atualmente denominado Programa Nacional de Controle do Tabagismo e Outros Fatores de Risco de Câncer (PNCTOFR $)^{5}$ e que é referência mundial. O objetivo geral do PNCTOFR é reduzir a prevalência de fumantes no Brasil e a consequente morbimortalidade por doenças relacionadas ao tabaco, com ações implementadas de forma descentralizada, utilizando o sistema de gerência do Sistema Único de Saúde (SUS) ${ }^{5-7}$.

Inquéritos nacionais, embora com metodologias distintas, têm evidenciado redução da prevalência do tabagismo. Em 1989, a Pesquisa Nacional de Saúde e Nutrição (PNSN) mostrou que $34,8 \%$ dos adultos eram tabagistas 7 . Já em 2002/ 2003, uma pesquisa nacional desenvolvida pelo Inca apontou prevalência de $22,4 \%$, o que representou queda de $35 \%$ em relação à $1989^{\circ}$. Em 2008, a Pesquisa especial de Tabagismo (PETab) apresentou $17,2 \%$ de fumantes 9 . E ainda, dados do VIGITEL (Vigilância de Fatores de Risco e Proteção para Doenças Crônicas por Inquérito Telefônico), estudo que fornece estimativas sobre a frequência e a distribuição sociodemográfica de fatores de risco e proteção para doenças crônicas não transmissíveis (DCNT), nas capitais dos 26 estados brasileiros e no Distrito Federal, no ano de 2006, revelou frequência de $16,2 \%$ de fumantes adultos e alcançou em 2011, 14,8\% ${ }^{10}$.

O Brasil está entre os países cujas políticas de controle do tabaco encontram-se em estágios avançados ${ }^{11}$. Entretanto, são raros os estudos que abordam a efetividade das diferentes ações propostas e executadas pelo PNCTOFR. Neste contexto, o presente estudo tem como objetivo apresentar as ações do PNCTOFR identificando as evidências de sua eficácia na redução da prevalência do tabagismo no Brasil.

\section{Métodos}

Realizou-se pesquisa bibliográfica sobre o PNCTOFR, utilizando-se os documentos disponíveis nos portais eletrônicos do Inca, da Política Nacional de Promoção da Saúde (PNPS), do Observatório da Política Nacional de Controle do Tabaco, da Organização Mundial da Saúde (OMS) e da Organização Pan-Americana de Saúde (OPAS). Estes documentos foram empregados na identificação das iniciativas nacionais para o controle do tabagismo. De forma complementar, foram efetuadas consultas às bases de dados Lilacs (Literatura Latino-americana e do Caribe), PubMed Central, Scielo (Scientific Eletronic Library Online) e ScienceDirect (Elsevier B.V.), sendo identificados estudos, disponíveis na íntegra, que apresentassem evidências da eficácia das ações do programa brasileiro de combate ao tabagismo.

A seleção dos descritores utilizados para consulta às bases de dados foi efetuada mediante consulta ao DEC (descritores de assunto em ciências da saúde da Bireme), sendo empregados os termos "Tabaco" e "Programa Nacional de Controle do Tabagismo" e seus respectivos correspondentes em inglês, combinados por meio dos operadores lógicos "AND" e "OR".

Foi realizada busca ativa de dados que pudessem refletir resultados das ações do PNCTOFR, bem como informações disponíveis no Datasus (Sistema de Informática do Sistema Único de Saúde) e no site do Ministério do Desenvolvimento Agrário (MDA). Para pesquisa de dados no Datasus, foram assumidas como doenças tabaco-relacionadas aquelas propostas pelo estudo Cancer Prevention Study II (CPS II) e traduzidas para o português por Pinto ${ }^{12}$.

Para organização e avaliação das possíveis evidências da eficácia das ações do PNCTOFR, utilizou-se como referencial as áreas/dimensões propostas pela International Union for Health Promotion and Education (IUHPE) ${ }^{13}$, que compreendem: 1) Ações de Regulação do Mercado; 2) Intervenções na Educação e na Informação Pública; 3) Intervenções na Atenção Primária à Saúde (APS); 4) Intervenções Locais; 5) Intervenções no Local de Trabalho; e 6) Intervenções com Enfoque na Comunidade. Para apresentar os possíveis resultados globais das ações do PNCTOFR, foi acrescentado o item 7) Possíveis Impactos da Redução da Prevalência de Tabagismo. 


\section{Resultados}

\section{Ações de Regulação do Mercado}

A primeira estratégia de combate ao fumo elencada pela $\mathrm{IUHPE}^{13}$, diz respeito à regulação do mercado. No Brasil, o PNCTOFR apresenta medidas neste âmbito (Quadro 1).

O Brasil destaca-se como o primeiro país a conseguir banir os descritores das embalagens, $o$ segundo a inserir os alertas com frases e imagens nos maços e um dos poucos a restringir a publicidade e a instituir veto à indústria de alimentos em relação à comercialização de produtos que simulem os derivados do tabaco bem como suas embalagens. Todas estas estratégias são propostas da Convenção-Quadro para Controle do Tabaco (CQCT) ${ }^{14}$. No Brasil, as imagens e frases de alerta sanitário são, periodicamente, renovadas de forma a atualizar a informação e revigorar a atenção do consumidor ${ }^{14,15}$.

O serviço telefônico, impresso nos maços, configura-se num meio de fácil acesso aos fumantes e não fumantes que desejam se beneficiar com as informações e aconselhamentos e representa uma ação de baixo custo ${ }^{2,16}$. Pesquisa realizada em 2002 por meio do serviço Disque Saúde
- Pare de Fumar evidenciou que, entre os 89.305 entrevistados, $80 \%$ eram fumantes, $92 \%$ apoiaram a medida de inserção das fotos nas embalagens e $90 \%$ conheceram o número do serviço pelos maços de cigarros ${ }^{15}$. Neste mesmo ano, estudo realizado pelo Instituto Datafolha sobre o impacto das advertências, com 2.216 participantes acima de 18 anos, mostrou que $76 \%$ dos entrevistados apoiaram a obrigatoriedade das imagens, $54 \%$ dos fumantes indagados mudaram de ideia sobre as consequências causadas pelo tabagismo na saúde e $67 \%$ dos fumantes disseram ter sentido vontade de deixar de fumar ${ }^{17}$.

A PETab ${ }^{9}$ demonstrou que $65 \%$ dos tabagistas com mais de 15 anos pensaram em deixar de fumar por causa do rótulo de advertência e as mulheres mostraram maior sensibilidade às mensagens que os homens $(67,2 \%$ versus $63,5 \%)$. Em estudo realizado em Pelotas, a análise da tendência do tabagismo de 2001 a 2010 evidenciou maior redução na prevalência entre as mulheres ${ }^{18}$, $\mathrm{o}$ que pode demonstrar que as ações de combate ao tabagismo são melhor apreendidas por elas.

Estudo realizado por Galduróz et al. ${ }^{19} \mathrm{com}$ 15.502 estudantes brasileiros, entre 11 e 18 anos, revelou queda significativa no uso de derivados do tabaco, de 32,7\%, em 1997 para 25,02\%, em

Quadro 1. As principais ações do PNCTOFR quanto à regulação do mercado

\begin{tabular}{|l|l|l|}
\hline \multicolumn{1}{|c|}{ Áreas } & \multicolumn{1}{c|}{ Açães } \\
\hline Fiscalização & $\begin{array}{l}\text { A regulamentação, o controle e a fiscalização de todos os produtos derivados do } \\
\text { tabaco por meio da Agência Nacional de Vigilância Sanitária (ANVISA); }\end{array}$ \\
\hline $\begin{array}{l}\text { Restrição à } \\
\text { disponibilidade }\end{array}$ & $\begin{array}{l}\text { Proibição da venda de derivados de tabaco a menores de 18 anos; } \\
\text { Alta carga de impostos sobre a comercialização de produtos derivados do tabaco; }\end{array}$ \\
\hline $\begin{array}{l}\text { Controle da } \\
\text { Comerção e }\end{array}$ & $\begin{array}{l}\text { Veto à propaganda comercial, inclusive Internet, bem como à distribuição de qualquer } \\
\text { tipo de amostra ou brinde e sua comercialização em estabelecimentos de ensino e } \\
\text { saúde; } \\
\text { Restrição da exposição dos produtos no interior dos pontos de venda, desde que } \\
\text { acompanhada com mensagem de advertência; } \\
\text { Interdição da associação do produto à prática de atividades esportivas e também a } \\
\text { sugestão de seu consumo em locais ou situações perigosas, abusivas ou ilegais; } \\
\text { Proibição do patrocínio de atividades culturais e esportivas internacionais; } \\
\text { Inserção de advertências sanitárias acompanhadas de fotos nos maços de cigarros, } \\
\text { acompanhadas do número do Disque Saúde - Pare de Fumar; } \\
\text { Proibição do uso de descritores como light e suave nas embalagens; } \\
\text { Proibição da comercialização de produtos alimentícios que simulem os derivados do } \\
\text { tabaco, bem como as suas embalagens; }\end{array}$ \\
\hline Outras & $\begin{array}{l}\text { Suspensão do financiamento à cultura do fumo em regime de parceria ou integração } \\
\text { com a indústria do tabaco por meio do crédito público do Programa de } \\
\text { Fortalecimento da Agricultura Familiar (PRONAF). }\end{array}$ \\
\hline
\end{tabular}


2004, sugerindo que esta redução seja consequência das políticas adotadas durante o período de estudo, como a proibição da publicidade e dos descritores, assim como a inserção das advertências com fotos. Entretanto, 31,3\% dos tabagistas com 15 anos e mais perceberam publicidade relacionada ao cigarro nos pontos de venda e $21,3 \%$ fora destes postos ou em eventos esportivos. Disto percebe-se que a lei era descumprida, e hoje, devido à proibição desta propaganda também nos postos de venda ${ }^{20}$, carece de maior fiscalização, especialmente em bares, botequins e restaurantes, que representam $53,8 \%$ dos pontos de aquisição de cigarros?.

Pantini et al. ${ }^{11}$ afirmam que a restrição da publicidade fez com que as marcas investissem em embalagens e nos pontos de venda de forma que estes se tornassem atrativos, envolventes e interferissem cada vez mais na decisão de compra dos consumidores. O ambiente restritivo imposto ao tabaco, certamente contribuiu para diminuir a exposição de crianças, adolescentes e jovens adultos à publicidade desse produto. No entanto, mesmo com legislação que proíbe o merchandising no ponto de venda ${ }^{20}$ é possível que a influência da exposição do produto sobre a iniciação e uso do tabaco ainda não esteja eliminada.

Recentemente, a Agência Nacional de Vigilância Sanitária (Anvisa) publicou a resolução RDC no 14/2012, que restringe o uso de aditivos em produtos derivados do tabaco. Esses aditivos são substâncias adicionadas intencionalmen- te para mascarar o gosto ruim da nicotina, disfarçar o cheiro desagradável, reduzir a porção visível da fumaça e diminuir a irritabilidade da fumaça para os não fumantes, com intuito principal de atrair novos consumidores. A Anvisa alerta que mais de $60 \%$ dos jovens ao experimentarem cigarros escolhem produtos com sabor ${ }^{21}, \mathrm{o}$ que configura aromatizantes e flavorizantes como armadilhas para este público.

O Brasil, mesmo com alta tributação (cerca de $76,3 \%$ sobre o preço final), possui um dos cigarros mais baratos do mundo, porque o preço de produção é baixo, tornando o produto acessível à população de menor renda. E, devido a diferentes taxas de impostos, especialmente na América Latina (Argentina e Uruguai - 67\%; Paraguai $-13 \%)$, o Brasil é vítima do mercado ilegal, sendo estimado que $35 \%$ do mercado brasileiro sejam oriundos do contrabando ${ }^{3,22}$. Além disso, o preço dos derivados do tabaco no Brasil é baixo por causa do reduzido custo da mão de obra e da alta produção a qual, mesmo em queda (Figura 1), é a segunda maior produção mundial.

Segundo dados do PETab ${ }^{9}$, a despesa média mensal com cigarros industrializados era de $\mathrm{R} \$$ 78,43 , o que representa $18,9 \%$ do salário mínimo vigente no ano da pesquisa. Com intuito de coibir a evasão tributária e recuperar a arrecadação do setor destinada ao tratamento de saúde dos consumidores de tabaco, foi fixado, por meio da sanção da lei no ${ }^{\circ} 2.546 / 11^{20}$, preço mínimo para comercialização dos cigarros em todo o território

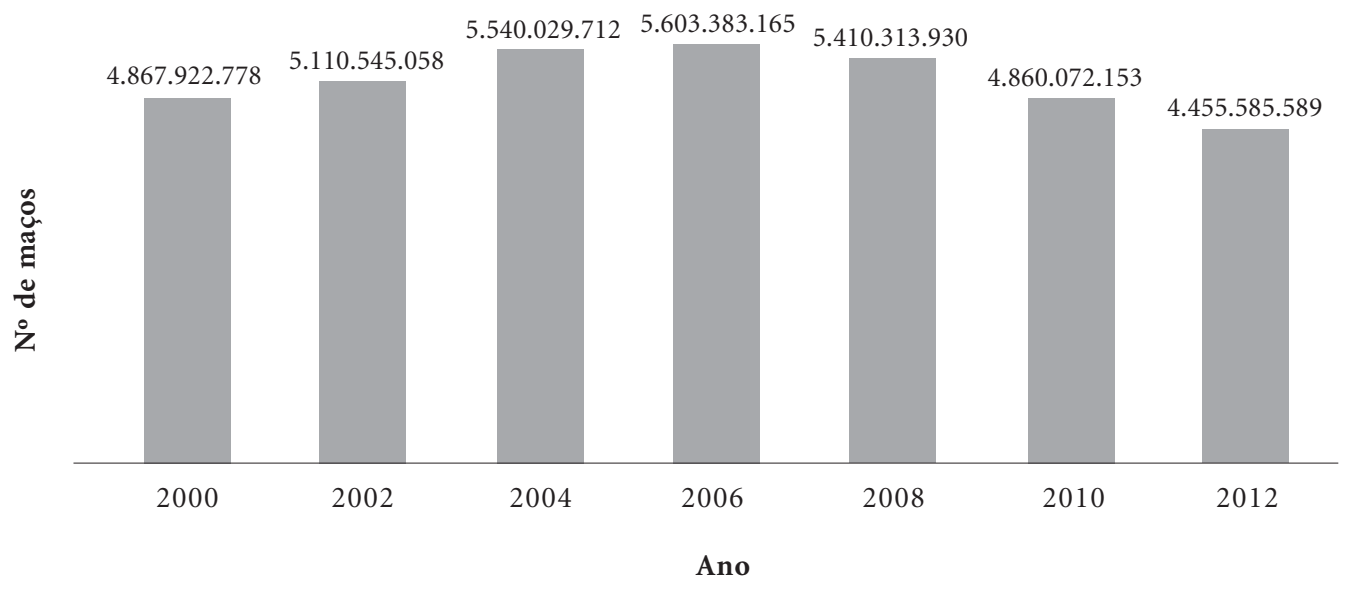

Figura 1. Produção de cigarros no Brasil, 2000 a 2012. 
nacional. Para vigência em 2013, o valor fixado para o maço (20 unidades) será de $R \$ 3,50$, o que representará maior comprometimento do salário para manutenção do consumo.

A suspensão do crédito público do Programa Nacional de Fortalecimento da Agricultura Familiar (Pronaf) para os produtores de fumo que mantém parceria com indústrias fumageiras representa desestímulo ao cultivo, já que o Pronaf destina-se ao apoio financeiro das atividades agropecuárias e não agropecuárias, a juros baixos ( 1,5 a $4,5 \%$ ao ano) e com longo prazo para reembol$\mathrm{so}^{24}$. O objetivo de dificultar o acesso a esse crédito rural é tornar a fumicultura menos atrativa, instigando os agricultores a diversificarem sua produção e reduzirem as áreas de cultivo do fumo. Todavia, se utilizada de forma isolada, essa política causa efeitos apenas a curto prazo, uma vez que a redução na oferta elevaria proporcionalmente o preço do produto, tornando o cultivo do fumo novamente favorável aos agricultores ${ }^{24,25}$.

Segundo o Anuário Estatístico do Crédito Rural $^{26}$, em 2001 foram assinados 70.162 contratos do Pronaf para a fumicultura, o que se reduziu para 801 em 2003, após a interrupção do custeio. Entretanto, em 2006, alcançou 1.325 contratos. E, de acordo com o Censo Agropecuário, as áreas brasileiras destinadas ao cultivo do fumo aumentaram quase $90 \%$ entre 1996 e $2006^{27}$, demonstrando que a suspensão do crédito não tem afetado a expansão das áreas de plantação do fumo e, consequentemente, o número de cigarros produzidos. Porém, não foram encontrados estudos nesta área. Em 2011, foram regidos 549 contratos do Pronaf para a fumicultura, acusando nova queda nos financiados ${ }^{26}$.

Entretanto, o grande desafio do Brasil, dos membros da CQCT, do movimento antifumo e dos ambientalistas é articular políticas de combate ao tabagismo com políticas de alternativas à fumicultura, combate à desigualdade social $\mathrm{e}$ degradação ambiental ${ }^{28}$. Neste sentido, aconteceu no Brasil em 2007, o primeiro seminário para o debate de questões referentes à continuidade $\mathrm{e}$ viabilização do Programa Nacional de Diversificação em Áreas Cultivadas com Tabaco. Baseado nos princípios do desenvolvimento sustentável, segurança alimentar, diversificação produtiva e participação social, o Programa atua na qualificação do processo de produção e de desenvolvimento nas áreas de plantio do fumo para cultivos alternativos ${ }^{25}$.

Desta forma o governo tenta reduzir a dependência em relação ao cultivo do fumo e viabilizar a reconversão produtiva a culturas diversas do fumo, sem prejuízos aos produtores. O MDA apoiou, até 2010, 60 projetos deste Programa em sete estados produtores de fumo, totalizando $\mathrm{R} \$$ 12 milhões investidos ${ }^{24}$. Fomentar ações do Programa Nacional de Diversificação em Áreas Cultivadas com Tabaco, visando ampliar sua cobertura é uma das propostas intersetoriais para a promoção da saúde e prevenção das DCNT indicadas no Plano de Ações Estratégicas para o Enfrentamento das DCNT no Brasil, 2011-202029.

\section{Intervenções na Educação e na Informação Pública}

No PNCTOFR intervenções na educação são estruturadas em ações pontuais e contínuas. Por meio da realização de atividades sistematizadas em subprogramas, o tema é inserido nas rotinas dos ambientes e instituições, alcançando o público-alvo dentro da sua realidade. O Programa "Saber Saúde" permite o desenvolvimento de ações preventivas para o controle do tabaco no ambiente escolar, com o objetivo de formar cidadãos críticos, capazes de fazer opções conscientes, na busca de melhor qualidade de vida. As informações e as atividades relacionadas ao consumo do tabaco e a outros fatores de risco de câncer são aplicadas ao cotidiano da escola, como parte do projeto pedagógico ${ }^{30}$.

Até o ano de 2009, foram capacitados, para execução do Programa "Saber Saúde", 122.214 professores de 14.419 escolas do Ensino Fundamental, alcançando 2.409.602 alunos. Neste mesmo ano foi estabelecida, oficialmente, a parceria com o Programa Saúde na Escola (PSE), o qual é uma política de articulação e integração entre as ações desenvolvidas nas escolas e nas Unidades Básicas de Saúde, em especial aquelas organizadas por meio da Estratégia de Saúde da Família (ESF). O objetivo principal do PSE é o de contribuir para o fortalecimento de ações que articulem saúde e educação para o enfrentamento dos fatores de risco que comprometem o pleno desenvolvimento de crianças e jovens brasileiros ${ }^{31}$.

Victora et al..$^{32}$ destacam o envolvimento concreto dos meios de comunicação com a saúde pública e ressaltam a contribuição quanto à moldagem do comportamento da população. A PETab ${ }^{9}$ mostrou que $67 \%$ dos fumantes com mais de 15 anos observaram informações anticigarro pela televisão ou rádio e, como formadores de opinião, estes meios de comunicação em massa contribuem para a desmistificação da imagem positiva do hábito de fumar e, assim, podem reduzir a atração, especialmente, de crianças e jovens pelos pro- 
dutos derivados do tabaco. Em datas evocativas como "Dia Mundial sem Tabaco" (31 de maio) e "Dia Nacional de Combate ao Fumo" (29 de agosto), o Inca tem elaborado e produzido materiais para serem distribuídos em campanhas, geralmente destacadas pela mídia, com objetivo de sensibilizar a população em geral ${ }^{31}$.

No Brasil, pesquisa realizada no município do Rio de Janeiro, evidenciou que a população estava bem informada, identificando elevado conhecimento sobre os riscos do tabaco em gestantes, que cigarros "light" não reduzem o risco de adoecimento, que a nicotina é droga e causa dependência, dentre outros aspectos ${ }^{33}$. Não obstante, muitas são as dificuldades a serem enfrentadas. Dados do Inquérito sobre Tabagismo entre escolares (Vigescola), envolvendo estudantes de 13 a 15 anos de idade em 12 capitais brasileiras, mostraram que a experimentação de cigarros entre os adolescentes variou de $31 \%$ a $58 \%$. O Vigescola também demonstrou que mais de $70 \%$ dos escolares compravam cigarros livremente ${ }^{34}$. Tais resultados demonstram a necessidade de intervenções direcionadas a este grupo populacional, bem como empoderamento da população para a desnormatização da venda de cigarros e similares para menores de 18 anos.

Além disso, o acesso à educação é crucial para redução do número de fumantes. A PETab ${ }^{9}$ mostrou que a prevalência do tabagismo é maior entre pessoas com menor escolaridade: menos de um ano - 25,7\%; 1 a 3 anos - 23,1\%; 4 a 7 anos$20,3 \%$; 8 a 10 anos - 14,9\%; 11 anos ou mais $11,9 \%$. Estudo de base populacional realizado em Pelotas (RS) demonstrou que a redução da prevalência de tabagismo entre aqueles pertencentes ao menor quintil de renda foi de $26 \%$, enquanto entre aqueles do maior quintil foi de $41 \%$ (p $<0,001)^{18}$.

\section{Intervenções na Atenção Primária à Saúde}

Considerando que cerca de $30 \%$ dos tabagistas brasileiros fumam 20 cigarros ou mais por dia, sugere-se que há elevado grau de dependência física da nicotina, o que requer suporte terapêutico ${ }^{8}$. Por meio do PNCTOFR, o MS/Inca tem investido em ações promotoras de mudanças de comportamento, preparando as unidades de saúde para oferecerem apoio efetivo à cessação de fumar aos tabagistas que procuram atendimentos de rotina na atenção primária ${ }^{30}$.

Com esse objetivo, foi desenvolvido o Programa "Unidades de Saúde Livres do Cigarro Saúde e Coerência" que envolve um conjunto de ações educativas, normativas e organizacionais, visando estimular mudanças culturais na aceitação social do livre consumo de derivados do tabaco em unidades de saúde e, num primeiro momento, apoiar os profissionais tabagistas a deixarem de fumar. Desta forma, o Programa inclui entre suas ações, a capacitação de profissionais de saúde para apoiarem a cessação de fumar dos funcionários da unidade de Atenção Primária à Saúde (APS), bem como a inserção deste apoio na rotina de atendimento à população ${ }^{30}$. Em 2009, 5.488 profissionais de saúde foram capacitados, representando aumento de $13 \%$ em relação ao ano anterior ${ }^{31}$.

Cerca de 57\% dos tabagistas com 15 anos ou mais foram advertidos a parar de fumar por serviços de saúde brasileiros ${ }^{35}$. Enquanto em 2005, 198 unidades de saúde realizavam o tratamento do tabagismo, até junho de 2009, 923 unidades atendiam os fumantes pelo SUS ${ }^{31}$. Segundo dados do Relatório de Gestão do Inca ${ }^{31}$, a programação de insumos para o ano de 2010, realizada pelos municípios, projetou aumento de $266 \%$ no número de municípios que executam programas de controle do tabagismo na APS.

No SUS, o tratamento do tabagismo é feito por meio da abordagem cognitivo-comportamental e apoio medicamentoso, quando indicado. A abordagem cognitivo-comportamental associa intervenções cognitivas com treinamento de habilidades comportamentais, sendo muito utilizada no tratamento das dependências ${ }^{36}$ (Quadro 2).

De acordo com o PETab 9 , 21,9\% dos fumantes fizeram tentativas de parar de fumar nos 12 meses anteriores à pesquisa, $15,2 \%$ por meio do aconselhamento e $6,7 \%$ pela farmacoterapia. Os medicamentos com eficácia comprovada na redução dos sintomas da síndrome de abstinência incluem os nicotínicos, dentre os quais destacamse o adesivo e a goma de mascar, utilizados no Brasil, e os não nicotínicos (antidepressivos) ${ }^{36,37}$. O suporte farmacológico associado ao aconselhamento cognitivo-comportamental foi destacado por pesquisadores como fundamental para a obtenção de 28,7\% de abstinentes após término do tratamento com grupo terapêutico, uma vez que mais de $80 \%$ desses indivíduos realizaram farmacoterapia ${ }^{38}$.

Estudo realizado no Brasil mostrou que o tratamento com adesivo de nicotina aumentou, aproximadamente, $50 \%$ a proporção de abstinência quando comparado a grupos controles, independentemente do número de sessões de aconselhamento ${ }^{39}$. Também foi constatado que 
Quadro 2. Principais abordagens cognitivo-comportamentais utilizadas no processo de cessação de fumar.

\begin{tabular}{|c|c|}
\hline $\begin{array}{c}\text { Abordagens } \\
\text { cognitivo-comportamentais }\end{array}$ & Características \\
\hline $\begin{array}{c}\text { Abordagem breve/mínima } \\
\text { (PAAP) }\end{array}$ & $\begin{array}{l}\text { Consiste em perguntar, avaliar, aconselhar e preparar o fumante para que } \\
\text { deixe de fumar, sem acompanhá-lo nesse processo. } \\
\text { Vantagens: realizada por qualquer profissional; estratégia de baixo custo. } \\
\text { Duração: } 3 \text { minutos }\end{array}$ \\
\hline Abordagem básica (PAAPA) & $\begin{array}{l}\text { Consiste em perguntar, avaliar, aconselhar, preparar e acompanhar o } \\
\text { fumante para que deixe de fumar. } \\
\text { Vantagens: realizada por qualquer profissional; prevê o retorno do } \\
\text { paciente para acompanhamento na fase crítica da abstinência; estratégia } \\
\text { de baixo custo. } \\
\text { Duração: } 3 \text { a } 5 \text { minutos }\end{array}$ \\
\hline $\begin{array}{c}\text { Abordagem específica/ } \\
\text { intensiva }\end{array}$ & $\begin{array}{l}\text { Atendimento em ambulatório específico para atender fumantes que } \\
\text { querem deixar de fumar. Individual ou em grupo. } \\
\text { Duração: no mínimo } 90 \text { minutos distribuídos em } 4 \text { sessões com } \\
\text { periodicidade semanal. }\end{array}$ \\
\hline
\end{tabular}

Fonte: Rosemberg ${ }^{37}$.

a associação da goma de nicotina com o adesivo nicotinado ou com antidepressivo elevou o número de sucessos na cessação de fumar ${ }^{37}$. Trabalho desenvolvido no Ambulatório de Auxílio ao Abandono do Tabagismo, no Rio Grande do Sul mostrou que os fumantes que receberam somente aconselhamento e terapia comportamental tiveram percentual de sucesso menor $(\mathrm{p}<0,05)$ do que os que receberam também alguma forma de tratamento farmacológico, evidenciando a importância desses medicamentos contra a dependência de nicotina. Neste estudo, 37,2\% dos pacientes fracassaram no tratamento ${ }^{40}$.

Para os tratamentos realizados na APS, pelo SUS, há relatos de $61,85 \%$ de cessação do tabagismo, em $2008^{31}$. Porém, não há dados sobre a evolução desses pacientes (sucesso ou recaídas). Estudo realizado com 160 participantes de Grupos de Apoio Terapêutico ao Tabagista (GATT) da APS do estado do Espírito Santo, nos quais era utilizada a abordagem cognitivo-comportamental, mostrou que, de nove a 20 meses após o término do tratamento, $28,7 \%$ dos indivíduos permaneciam abstinentes, 51,9\% recaíram e 19,4\% não pararam de fumar ${ }^{38}$. Ainda que a adesão dos indivíduos aos grupos terapêuticos se reduza com o avançar do tratamento ${ }^{41}$, a formação de grupos terapêuticos antitabagismo na APS, com participação multiprofissional representa uma alternativa de apoio e, consequentemente, de êxito na tentativa de abandono do tabaco ${ }^{42}$. Segundo Ramos et al. ${ }^{41}$, a redução do número de participan- tes ao longo do tratamento é previsto e alertado durante as capacitações pois, já na primeira sessão, o indivíduo é confrontado quanto à sua real intenção de abandonar o tabaco. Avalia-se que os que permanecem até o último encontro estão efetivamente motivados a parar de fumar, mesmo que não consigam fazê-lo no período previsto ${ }^{41}$. A saúde mental dos indivíduos que ingressam nesses grupos terapêuticos deve ser avaliada e, particularmente, considerada no tratamento, o que pode aumentar as taxas de abstinência, visto que ansiedade e depressão são quadros comuns entre essas pessoas ${ }^{38}$.

\section{Intervenções Locais}

A fumaça do tabaco liberada para o ambiente e que as pessoas inalam involuntariamente é conhecida como poluição tabagística ambiental (PTA) e o processo de respirar esta fumaça é denominado de tabagismo passivo ${ }^{43}$. No Brasil, a lei $n^{\circ}$ 9.294/1996 institui que o uso de cigarros ou qualquer produto fumígero, deve ser restrito à área destinada exclusivamente a esse fim (os chamados fumódromos) em recinto coletivo, seja privado ou público ${ }^{44}$, o que não limitava $100 \%$ a exposição. Assim, estados e municípios têm se adiantado e instituído leis que proíbem o uso de tabaco em ambientes públicos fechados, como nos estados do Rio de Janeiro (Lei 5.517/09) ${ }^{45} \mathrm{e}$ São Paulo (Lei 13.541/09) ${ }^{46}$ com intuito, especialmente, de reduzir a exposição à PTA. 
No entanto, após longa tramitação do Projeto de Lei no 315 e esforço do Inca, em 2011, a Lei Federal $n^{\circ} 12.546^{20}$ definiu a proibição total do fumo em ambientes fechados de uso coletivo, privados ou públicos. Cabe à Anvisa a fiscalização para que a lei seja cumprida e a população pode (e deve) contribuir com esta ação, a qual responde às recomendações da Organização Mundial de Saúde ${ }^{31}$.

Em 2006, a Gerência de Produtos Derivados do Tabaco (GPDTA) promoveu cinco oficinas regionais sobre os malefícios do fumo e o direito dos não fumantes a ambientes livres da fumaça do tabaco, das quais participaram representantes de todos os estados da União. Os resultados obtidos em consulta pública, realizada em 2007, demonstraram o interesse da população em garantir seus direitos a um ambiente menos poluído, uma vez que $71,06 \%$ manifestou-se a favor de restrição ao fumo em ambientes fechados ${ }^{47}$.

A poluição PTA nos domicílios, no trabalho e nos locais de lazer concorrem para os fracassos, assim como a nicotino-dependência e as pressões sociais ${ }^{37}$.

\section{Intervenções no Local de Trabalho}

A fumaça do tabaco é a maior fonte de poluição em ambientes fechados. O fato de que a maioria das pessoas passa $90 \%$ do seu tempo nesses ambientes, principalmente, em casa e no trabalho, torna evidente a gravidade dessa exposição $0^{48}$.

No Brasil, o PNCTOFR atua nos ambientes de trabalho, por meio do módulo específico "Prevenção Sempre”, cujo objetivo é atuar junto a profissionais da área de saúde ocupacional e outros atores-chave das organizações empresariais a fim de tornar o ambiente de trabalho livre da PTA e reduzir o número de funcionários tabagistas. Este módulo visa estimular mudança de comportamento em relação ao tabagismo entre os trabalhadores com a realização de ações educativas, além de ações normativas e organizacionais ${ }^{3,5}$.

Pesquisa realizada com objetivo de avaliar o impacto da lei antifumo na cidade de São Paulo analisou a concentração de monóxido de carbono (CO) em 585 restaurantes, bares e similares, em diferentes ambientes (fechados, semiabertos e áreas internas) e no ar expirado por 627 trabalhadores destes locais. As medições foram realizadas antes e 12 meses após implementação da lei. Foi encontrada redução de 70\% na concen- tração de $\mathrm{CO}$ nos estabelecimentos e também no ar expirado pelos trabalhadores, independente de serem estes fumantes ou não ${ }^{49}$.

Dados da PETab ${ }^{9}$ revelam que, no Brasil, em 2008, os locais de trabalho foram apontados como o segundo lugar de maior exposição à PTA $(24,4 \%)$, o que justifica a importância do combate ao tabagismo nestes locais como parte das políticas de saúde ${ }^{35}$. Além disso, o direito dos trabalhadores à redução dos riscos para a saúde presentes no trabalho está inscrito no artigo $7^{\circ}$ da Constituição Federal de $1988^{50}$ e a lei no $12.546 /$ $11^{20}$ vai ao encontro dessa disposição legal.

\section{Intervenções com Enfoque na Comunidade}

O PNCTOFR tem como base de atuação a utilização do sistema de gerência do SUS estando, portanto, inserido no contexto da ESF, que prioriza ações de proteção e promoção à saúde dos indivíduos e da família e envolve o conhecimento da realidade, a identificação de problemas e a elaboração de planejamento em nível de comunidade. Assim, o PNCTOFR é um programa cujo enfoque de atuação está baseado na comunidade e a diminuição da aceitação social do tabagismo dentre outros resultados positivos encontrados no Brasil apresentam estreita relação com este método ${ }^{51}$.

A implementação da Lei Antifumo no estado de São Paulo obteve grande sucesso. Em pesquisa telefônica realizada pelo governo do estado um mês após a vigência da lei, foi encontrado $94 \%$ de apoio da população à proibição do fumo em ambientes fechados. Mesmo em curto período de tempo, este estudo demonstrou aumento em torno de 30\% na procura por tratamentos para deixar de fumar. Em resposta, o governo anunciou a ampliação de programas de capacitação para o tratamento do tabagismo, passando a incluir as 3.100 equipes da ESF estaduais ${ }^{52}$.

Em 2009, o Inca em parceria com a Aliança para o Controle do Tabagismo, ACT-br, inaugurou o projeto 'Fala Sério!', voltado para o público universitário. A ideia do 'Fala Sério' foi introduzir nas universidades brasileiras um debate sobre as diferentes formas de atuação da indústria do tabaco, utilizando como ferramenta distintas formas de ação cultural (filmes, exposições, debates, etc), a partir de um ponto de vista regional ${ }^{31}$. 


\section{Possíveis Impactos da Redução na Prevalência de Tabagismo}

A OMS estima que o fumo seja responsável por, aproximadamente, $70 \%$ dos cânceres de pulmão, $42 \%$ das doenças respiratórias crônicas e $10 \%$ das doenças do aparelho circulatório ${ }^{31}$. A redução do tabagismo pressupõe a diminuição na prevalência das doenças tabaco-relacionadas. Com intuito de obter esta informação, foi realizada busca ao Datasus das doenças relacionadas ao tabaco pelo estudo CPS II e traduzidas para o português ${ }^{12}$. As doenças foram agrupadas em três grandes grupos: Doenças do Aparelho Circulatório (DAC), Doenças do Aparelho Respiratório (DAR) e Cânceres (Figura 2). De acordo com a figura, houve aumento na taxa de mortalidade pelos três grupos, especialmente, a partir de 2000.

Assim, a redução da prevalência do tabagismo não se refletiu na diminuição da mortalidade por doenças tabaco-relacionadas. Por serem dados secundários, podem ter contribuído para este fato: a melhora na capacidade diagnóstica, devido ao maior acesso aos serviços de saúde; aumento na notificação; bem como a estrutura etária da população, a qual está envelhecendo e tem sido mais acometida por doenças crônicas, especialmente DAC. Além disso, as doenças associadas ao tabaco apresentam um período para se manifestarem. Desta forma, a elevação da mortalidade mostrada de 1996 até 2010 pode, também, ser reflexo da elevada prevalência de tabagismo no Brasil na década de 80 e anteriores, bem como do aumento dos demais fatores de risco, como obesidade e inatividade física ${ }^{29}$.

Todavia, o estudo de Schmidt et al. ${ }^{53}$ foi capaz de mostrar redução da mortalidade por DCNT, de 1996 a 2007. Os pesquisadores padronizaram os óbitos por idades para a populaçãopadrão, segundo a OMS, e corrigiram os dados para sub-registro e causas mal definidas. De acordo com os autores, a mortalidade atribuível às DCNT diminuiu, sobretudo, devido à redução nas DAC e DAR. Para os autores, não está claro até que ponto essas tendências seculares se devem a melhorias no acesso à atenção à saúde, à diminuição do tabagismo ou outras causas.

Estudo que analisou a mortalidade por câncer no Brasil, mostrou que a taxa de óbitos por cânceres relacionados ao tabaco encontrava-se estabilizada até $1995^{54}$. Já Malta et al..$^{55}$ observaram diminuição na mortalidade por câncer de pulmão até 2003, o que foi atribuído à queda na prevalência do tabagismo.

Presume-se o ônus do tabagismo para uma população pela redução da qualidade de vida, tanto pelo gasto com a compra de produtos derivados do tabaco, quanto pela diminuição da produtividade, especialmente da população em idade economicamente ativa, além dos gastos do siste-

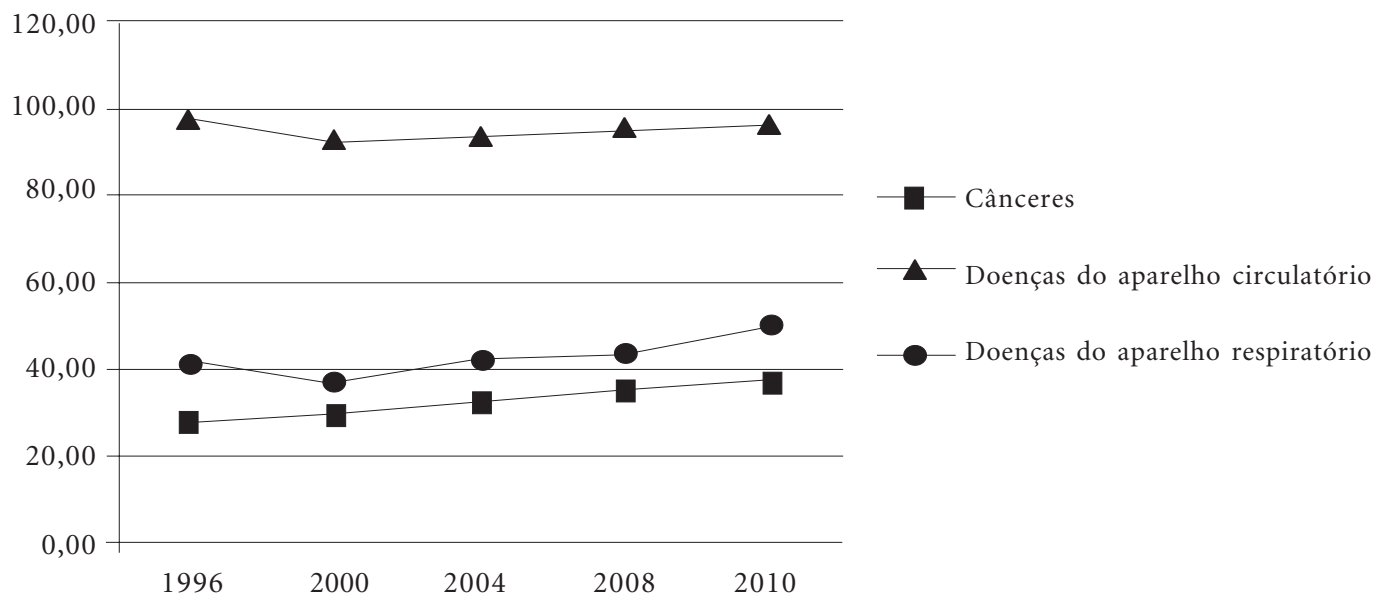

Figura 2. Taxa de Mortalidade Geral por 100.000 habitantes de grupos de doenças tabaco-relacionadas, segundo proposta de Pinto ${ }^{12}$, num período de 1996 a 2010. 
ma público de saúde com a morbidade tabacorelacionada. Pesquisa apontou que 35,9\% das internações de homens e $27 \%$ das internações de mulheres foram atribuíveis ao tabagismo, em 2005. E, do montante de $\mathrm{R} \$ 3.809 .446 .536,23$ de custos de internações de pessoas com mais de 35 anos, 6,9\% foram atribuíveis ao hábito de fu$\operatorname{mar}^{56}$. Os autores reiteram que, apesar de os resultados serem relativos e, possivelmente, subestimados, indicam a carga econômica do tabagismo sobre o SUS e pode ser apenas a "ponta do iceberg" dos custos reais.

\section{Discussão}

O Global status report on noncommunicable diseases 2010, da $\mathrm{OMS}^{57}$, ressalta o reforço às proibições e advertências para promoção do tabaco e seus derivados, o aumento dos impostos e a restrição ao acesso a esses produtos como ações que podem resultar em imediata prevenção de doenças e redução de custos em saúde. O PNCTOFR tem exercido essas ações e, como demonstrado por Schmith et al. ${ }^{53}$, as doenças tabacorelacionadas têm reduzido o que deve ocasionar diminuição nos gastos. E, o princípio norteador das proposições da CQCT é que "toda pessoa deve ser informada sobre as consequências sanitárias impostas pelo consumo e exposição à fumaça do tabaco e medidas legislativas, executivas, administrativas e outras ações efetivas devem ser implementadas em nível governamental para proteger todas as pessoas da exposição à fumaça do tabaco" ${ }^{58}$, o que podemos ver nas ações de regulação do mercado e nos projetos e programas desenvolvidos pelo PNCTOFR, como "Fala Sério" e "Unidades de Saúde Livres do Cigarro - Saúde e coerência”, direcionados, respectivamente, à informação de universitários e profissionais de saúde e população em geral.

$\mathrm{O}$ veto à propaganda, a exposição dos produtos limitada aos pontos de venda acompanhada de mensagens de advertência, a proibição de sua associação a atividades esportivas e culturais, e, ainda, o impedimento ao patrocínio de eventos diversos, busca inibir a figura positiva e inofensiva atribuída, pelas indústrias tabageiras, ao hábito de fumar. No entanto, ainda é necessária maior rigidez quanto à exibição feita nos postos de venda, a qual continua atrativa e, muitas vezes, de fácil acesso ao público infanto-juvenil. Para a OMS, o tabagismo é uma doença pediátrica, uma vez que cerca de $90 \%$ dos fumantes regulares iniciam antes dos 18 anos de idade ${ }^{15}$. A proibição da publicidade de cigarros é ameaça às vendas reconhecida pelas próprias indústrias fumageiras ${ }^{1,59,60}$ e as proibições totais da publicidade potencializam as demais intervenções ${ }^{2}$ e precisam ser legitimadas pelo PNCTOFR.

A inserção das imagens de advertência nas embalagens reforça a proibição da publicidade e torna a informação clara, independente da escolaridade, e destacada ao consumidor, além de atuar como recurso para motivação da mudança de comportamento visando à redução do consumo e da iniciação ao uso ${ }^{2,14,15}$. É definido em lei que $100 \%$ da face posterior das embalagens de produtos fumígeros seja ocupada com cláusulas de advertência legíveis e destacadas ostensivamente. A partir de 2016, 30\% da parte frontal inferior das embalagens, também, apresentará texto de advertência ${ }^{20}$.

Há consenso entre os pesquisadores de que a medida isolada mais efetiva para o controle do tabagismo é a alta taxa de impostos ${ }^{2,37,59,61,62}$. No entanto, a elevação de impostos pode estimular o mercado ilegal, como ocorre na América do Sul. Segundo o Banco Mundial ${ }^{57}$, o contrabando não é decorrente apenas de impostos e preços elevados. A corrupção é o fator explicativo. O Banco Mundial sugere que, a elevação média de $10 \%$ no preço dos cigarros incrementa em torno de $10 \%$ as receitas, reduz cerca de $3,5 \%$ o consumo e aumenta discretamente o contrabando ${ }^{57}$, o qual deve ser combatido veementemente pelos países. O empoderamento da população, assim como uma coordenação internacional, contribuem para este combate.

Para Wünsch Filho et al. ${ }^{63}$, hospitais e escolas são espaços críticos para a política de ambientes livres da fumaça do tabaco, pois os profissionais de saúde, assim como os professores e trabalhadores em educação, podem irradiar modelos de comportamento, particularmente, para os adolescentes e jovens. O projeto "Fala Sério" e os programas "Saber Saúde", Saúde na Escola e "Unidades de Saúde Livres do Cigarro - Saúde e Coerência" vão ao encontro dessas recomendações.

A abordagem a todos os indivíduos em nível de APS visa não apenas diagnosticar o tabagismo e aconselhar os fumantes, mas estimular aqueles que não fumam para que não comecem a fumar ${ }^{64}$ e que combatam a PTA. A promoção de ambientes livres de tabaco está entre as seis medidas indicadas pela OMS como prioritárias para conter a expansão do tabagismo e suas graves consequências para a saúde ${ }^{2}$ e o programa "Prevenção Sempre" procura sensibilizar e envolver empresários e instituições, com preparo 
prévio da direção e condições de sustentabilidade do programa por tempo suficiente ${ }^{65}$. A proibição do tabagismo no local de trabalho, especialmente, reduz as oportunidades de fumar e configura como estímulo ao abandono da prática.

A adesão e a aprovação, especialmente, da lei antifumo federal pela população são de importância peculiar para que sua implementação seja realizada com sucesso. A implementação de políticas que promovam ambientes livres do fumo é tendência mundial, fundamentada no conhecimento dos males provocados pela exposição à $\mathrm{PTA}^{66}$. Desta forma, o empoderamento da população e a participação social, princípios da promoção da saúde, são imprescindíveis para que a legislação seja efetivada. A existência de legislação que controle o consumo de tabaco em locais de trabalho é fator indispensável para a promoção da saúde, posto que elimina os riscos da exposição, além de apoiar os tabagistas que querem abandonar o vício, diminuindo a prevalência de fumantes em até $30 \%{ }^{49,67,68}$.

A eficácia de abordagens baseadas na comunidade foi comprovada em alguns programas internacionais que resultaram em redução de $10 \%{ }^{69,70}, 13 \%{ }^{70}$ e $30,6 \%{ }^{71,72}$ na prevalência de fumantes. No Brasil, não há dados sobre os resultados diretos das intervenções baseadas na comunidade que, possivelmente, muito contribuíram para diminuição da prevalência de fumantes no país.

Para Romero e Silva ${ }^{22}$ as informações estatísticas e epidemiológicas são suficientes para monitoramento da atuação governamental e da situação de controle das ações implementadas. No entanto, o resultado individual ou a contribuição de cada uma das diversas ações para a redução da prevalência do tabagismo no Brasil ainda é pouco investigado. O Inca tem incentivado as pesquisas em parcerias com universidades e entidades nacionais e internacionais, com intuito de monitorar e informar a situação do controle do tabagismo no país ${ }^{31}$.

A epidemia tabágica no país apresenta distribuição desigual entre as classes sociais, atingindo de forma mais intensa as populações de menor renda e com menor tempo de educação formal, logo, mais permeáveis às mensagens da indústria do tabaco, sem crivo crítico ${ }^{8,63,65}$. O sucesso do programa brasileiro de controle do tabagismo deve-se ao cumprimento das principais me- tas definidas em $1988^{22}$. Todavia, novas metas precisam ser estabelecidas, bem como inseridas novas propostas, como a regulamentação dos produtos de tabaco e de suas embalagens e publicidade, o controle do contrabando ${ }^{11,23}$, o apoio a alternativas ao cultivo do tabaco ${ }^{22}$ e ações de maior impacto sobre a população de menor renda e escolaridade, atualmente maiores contribuintes para prevalência de tabagismo no Brasil. Muitas dessas propostas foram previstas no Plano de Ações para Enfrentamento das DCNT no Brasil para 2011 a 2020 e são defendidas para o alcance da projeção de $0,3 \%$ de queda anual do tabagismo em adultos ${ }^{29}$. A lei no $12.546 / 11^{20}$ já apresenta avanços, particularmente, quanto à propaganda e tributação, que contribuirão para o cumprimento desta nova meta.

Um país com a epidemia do tabagismo controlada, também significa redução dos casos de doenças associadas ao tabaco, menores gastos para o SUS, melhor qualidade de vida para os fumantes passivos, redução do número de adolescentes fumantes e da mortalidade precoce, dentre outros inúmeros benefícios ${ }^{11}$.

\section{Considerações Finais}

Os resultados deste estudo apontam para a eficácia do PNCTOFR. Contudo, apesar da abordagem inovadora deste e da redução da prevalência e incidência do tabagismo no Brasil, o hábito de fumar ainda é um grande problema de saúde pública, o qual exige atenção e vigilância constante das políticas e controle social para a desnormatização desse comportamento. Além disso, novas investigações sobre os resultados das ações deste programa brasileiro permitirão identificar lacunas na sua atuação, além da reorientação e do reforço às ações, potencializando e inovando suas várias estratégias.

Não obstante, é importante destacar o menor custo das políticas de promoção da saúde direcionadas ao combate ao tabagismo comparado aos gastos públicos dispensados ao tratamento das DCNT associadas ao tabaco, justificando a relevância de se manter, implementar e até mesmo ampliar as ações do PNCTOFR, a fim de se obter resultados cada vez mais favoráveis, especialmente para os grupos populacionais mais vulneráveis ao tabagismo. 


\section{Colaboradores}

ST Silva, MC Martins, FR Faria, RMM Cotta participaram igualmente de todas as etapas de elaboração do artigo.

\section{Referências}

1. Organização Pan-Americana de Saúde (Opas), Organização Mundial da Saúde (OMS). Prevención y Control del Consumo de Tabaco. Washington: OMS, OPAS; 1998. 122 Sessão.

2. Organização Mundial da Saúde, Organização PanAmericana de Saúde. Who report on the global tobacco epidemic, 2008: The MPOWER package. Geneva: OMS; 2008.

3. Brasil. Ministério da Saúde (MS). Diretoria de Prevenção e Vigilância, Instituto Nacional do Câncer. Programa Nacional de Controle do Tabagismo e Outros Fatores de Risco de Câncer. Modelo Lógico e Avaliação. Rio de Janeiro: Inca; 2003.

4. Cavalcante TM. Programa de controle do tabagismo no Brasil: avanços e desafios Brasil. Rev psiquiatr clin 2005; 32(5):283-300.

5. Brasil. Ministério da Saúde (MS). Instituto Nacional de Câncer (Inca). Coordenação de Prevenção e Vigilância - CONPREV. Programa Nacional de Controle do Tabagismo e outros Fatores de Risco - Brasil. Rio de Janeiro: Inca; 2001.

6. Brasil. Ministério da Saúde (MS). Portaria GM/MS/ N. ${ }^{\circ} 1.575$, de 29 de agosto de 2002. Consolida o Programa Nacional de Controle de Tabagismo, e dá outras providências. Diário Oficial da União 2002; 29 ago.

7. Instituto Nacional do Câncer (Inca). Programa Saber Saúde: buscando formar cidadãos conscientes. Atualidades em Tabagismo e Prevenção do Câncer 2001; 10 (jan./mar.).

8. Monteiro CA, Cavalcante TM, Moura EC, Claro RM, Szwarcwald CL. Population-based evidence of a strong decline in the prevalence of smokers in Brazil (1989-2003). Bull World Health Organ 2007; 85(7):527-534.

9. Brasil. Ministério do Planejamento, Orçamento e Gestão. Instituto Brasileiro de Geografia e Estatística (IBGE). Diretoria de Pesquisas. Coordenação de Trabalho e Rendimento. Pesquisa Nacional por Amostra de Domicílios - Tabagismo 2008. Rio de Janeiro: IBGE; 2009.

10. Portal da Saúde SUS. Número de fumantes segue em queda no Brasil. [página na Internet]. [acessado 2013 set 5]. Disponível em: http://portal.saude.gov.br/ portal/aplicacoes/noticias/default.cfm?pg =dsp DetalheNoticia\&id_area=124\&CO_NOTICIA=13839.

11. Pantani D, Pinsky I, Monteiro A. Publicidade de tabaco no ponto de venda. São Paulo: Ed. do Autor; 2011.
12. Pinto MFT. Custos de doenças tabaco-relacionadas: uma análise sob a perspectiva da economia e da epidemiologia [tese]. Rio de Janeiro: Escola Nacional de Saúde Pública; 2007.

13. Unión Internacional de Promoción de la Salud y Educación para la Salud (IUHPE). La Evidencia de la Eficacia de la Promoción de la Salud. Parte Dos. St. Denis Cedex: IUHPE; 1999.

14. Brasil. Ministério da Saúde (MS). Agência Nacional de Vigilância Sanitária (Anvisa). A Anvisa e o controle dos produtos derivados do tabaco. Brasília: MS; 2007.

15. Brasil. Ministério da Saúde (MS). Diretoria de Prevenção e Vigilância. Instituto Nacional do Câncer (Inca). Brasil: advertências sanitárias nos produtos de tabaco 2009. Rio de Janeiro: Inca; 2008.

16. Miller CL, Wakefield M, Roberts L. Uptake and effectiveness of the Australian telephone Quitline service in the context of a mass media campaign. Tob Control 2003; 12(Supl. 2):53-58.

17. Datafolha. Opinião Pública. São Paulo: Datafolha, 21 abr. 2002. [página na Internet]. [acessado 2013 set 5]. Disponível em: http://datafolha.folha.uol. com.br/po/ver_po.php?session=625.

18. Dias-Damé JL, Cesar JÁ, Silva SM. Tendência temporal de tabagismo em população urbana: um estudo de base populacional no Sul do Brasil. Cad Saude Publica 2011; 27(11):2166-2174.

19. Galduróz JCF, Fonseca AM, Noto AR, Carlini EA. Decrease in tobacco use among Brazilian students: A possible consequence of the ban on cigarette advertising? Addict Behav 2007; 32(6):1309-1313.

20. Brasil. Presidência da República. Lei no 12.546 , de 14 de dezembro de 2011. Institui o Regime Especial de Reintegração de Valores Tributários para as Empresas Exportadoras (Reintegra); dispõe sobre a redução do Imposto sobre Produtos Industrializados (IPI) à indústria automotiva; altera a incidência das contribuições previdenciárias devidas pelas empresas que menciona; altera as Leis no 11.774 , de 17 de setembro de 2008, no 11.033 , de 21 de dezembro de 2004, no 11.196 , de 21 de novembro de 2005, no 10.865, de 30 de abril de 2004, no 11.508, de 20 de julho de 2007, no 7.291, de 19 de dezembro de 1984, no 11.491, de 20 de junho de 2007, no 9.782, de 26 de janeiro de 1999, e no 9.294, de 15 de julho de 1996, e a Medida Provisória no 2.199-14, de 24 de agosto de 2001; revoga o art. 1o da Lei no 11.529 , de 22 de outubro de 2007 , e o art. 60 do Decreto-Lei no 1.593, de 21 de dezembro de 1977, nos termos que especifica; e dá outras providências. Diário Oficial da União 2011; 14 dez. 
21. Brasil. Agência Nacional de Vigilância Sanitária. Resolução no 14 , de 15 de março de 2012. Dispõe sobre os limites máximos de alcatrão, nicotina e monóxido de carbono nos cigarros e a restrição do uso de aditivos nos produtos fumígenos derivados do tabaco, e dá outras providências. Diário Oficial da União 2012; 16 mar.

22. Romero LC, Costa e Silva VL. 23 Anos de Controle do Tabaco no Brasil: a Atualidade do Programa Nacional de Combate ao Fumo de 1988. Rev Bras Cancerol 2011; 57(3):305-314.

23. Brasil. Receita Federal. Produção de Cigarros no Brasil. [página na Internet]. 2012 [acessado 2013 set 5]. Disponível em: http://www.receita.fazenda.gov.br/ DestinacaoMercadorias/ProgramaNacCombCigarro Ilegal/Producao_cigarros_historico.htm.

24. Brasil. Ministério do Desenvolvimento Agrário. Projetos Especiais - Programa Nacional de Diversificação em Áreas Cultivadas com Tabaco. [página na Internet]. [acessado 2013 set 5]. Disponível em: http:/ /www.mda.gov.br/portal/saf/programas/projetos especiais/2308129

25. Brasil. Ministério do Desenvolvimento Agrário. Relatório - $1^{\circ}$ Seminário do programa de diversificação em áreas cultivadas com tabaco. Brasília: Ministério do Desenvolvimento Agrário; 2007.

26. Brasil. Banco Central do Brasil. Anuário Estatístico do Crédito Rural. [página na Internet]. 2011 [acessado 2013 set 5]. Disponível em: http://www. bcb. gov.br/?RELRURAL

27. Brasil. Instituto Brasileiro de Geografia e Estatística (IBGE). Censo Agropecuário. [página na Internet]. 2006. [acessado 2013 set 5]. Disponível em: http:// www.ibge.gov.br/home/estatistica/economia/agropecuaria/censoagro/brasil_2006/default.shtm

28. Brasil. Ministério da Saúde (MS). Secretaria de Vigilância em Saúde. Secretaria de Atenção à Saúde. Política Nacional de Promoção da Saúde. $3^{a}$ Edição. Série B. Textos Básicos de Saúde, Série Pactos pela Saúde 2006. Brasília: MS; 2010.

29. Brasil. Ministério da Saúde (MS). Secretaria de Vigilância em Saúde. Departamento de Análise de Situação de Saúde. Plano de ações estratégicas para o enfrentamento das doenças crônicas não transmissíveis (DCNT) no Brasil 2011-2022. Brasília: MS; 2011. (Série B. Textos Básicos de Saúde)

30. Brasil. Ministério da Saúde (MS). Instituto Nacional do Câncer (Inca). Coordenação de Prevenção e Vigilância - CONPREV. Programa Nacional de Controle do Tabagismo e outros Fatores de Risco - Brasil. Rio de Janeiro: Inca, 2001.

31. Instituto Nacional do Câncer (Inca). Relatório de Gestão - Exercício 2009. Rio de Janeiro: Inca; 2009.

32. Victora CG, Barreto ML, Leal MC, Monteiro CA, Schmidt MI, Paim J, Bastos FI, Almeida C, Bahia L, Travassos C, Reichenheim M, Barros FC, the Lancet Brazil Series Working Group. Condições de saúde e inovações nas políticas de saúde no Brasil: o caminho a percorrer. Lancet Série Brasil 2011; 90-102.

33. Brasil. Ministério da Saúde (MS). Instituto Nacional do Câncer (Inca). Programa Nacional de Controle do Tabagismo e outros Fatores de Risco de Câncer. Modelo Lógico e Avaliação. 2a Edição. Rio de Janeiro: Inca; 2005.
34. Brasil. Ministério da Saúde (MS). Secretaria de Atenção à Saúde. Instituto Nacional de Câncer (Inca). Coordenação de Prevenção e Vigilância (CONPREV). Vigescola. Vigilância de tabagismo em escolares: dados e fatos de 12 capitais brasileiras. Rio de Janeiro: Inca; 2004.

35. Instituto Brasileiro de Geografia e Estatística (IBGE). Pesquisa Nacional por Amostra de Domicílios. Rio de Janeiro: IBGE; 2008.

36. Brasil. Ministério da Saúde (MS). Secretaria de Assistência à Saúde. Instituto Nacional de Câncer (Inca). Abordagem e tratamento do fumante: consenso. Rio de Janeiro: Inca; 2001.

37. Rosemberg J. Nicotina Droga Universal. [página na Internet]. 2003. [acessado 2013 set 5]. Disponível em: http://www.inca.gov.br/tabagismo/publicacoes/ nicotina.pdf

38. Sattler AC, Cade NV. Prevalência da abstinência ao tabaco de pacientes tratados em unidades de saúde e fatores relacionados. Cien Saude Colet 2013; 18(1): 253-264.

39. Otero UB, Perez CA, Szklo M, Esteves GA, Pinho MM, Szklo AS, Turci SRB. Ensaio clínico randomizado: efetividade de abordagem cognitivo-comportamental e uso de adesivos transdérmicos de reposição de nicotina, na cessação de fumar, em adultos residentes no município do Rio de Janeiro, Brasil. Cad Saude Publica 2006, 22(2):439-449.

40. Haggsträm FM, Chatkin JM, Cavalet-Blanco D, Rodin V, Fritscher CC. Tratamento do tabagismo combupropiona e reposição nicotínica. J Pneumol 2001; 27(5):255-261.

41. Ramos D, Soares TST, Viegas K. Auxiliando usuários de uma unidade de saúde a parar de fumar: relato de experiência. Cien Saude Colet 2009; 14(Supl. 1):1499-1505

42. Veloso NS, Rodrigues CAQ, Leite MTS, Larissa J, Ottoni M, Veloso GCC, Rodrigues RM, Bandeira GA. Tabagismo: a percepção dos fumantes em um grupo de educação em saúde. Rev bras med fam comunidade 2011; 6(20):193-198.

43. Brasil. Ministério da Previdência Social. Política Nacional de Segurança e Saúde do Trabalhador. Brasília: Ministério da Previdência Social; 2004.

44. Brasil. Agência Nacional de Vigilância Sanitária. Lei no 9.294, de 15 de julho de 1996. Dispõe sobre as restrições ao uso e à propaganda de produtos fumígeros, bebidas alcoólicas, medicamentos, terapias e defensivos agrícolas, nos termos do $\$ 4^{\circ}$ do art. 220 da Constituição Federal. Diário Oficial da União 1996; 16 jul.

45. Rio de Janeiro. Assembléia Legislativa do Estado do Rio de Janeiro. Lei no 5.517 de 17 de agosto de 2009. Proíbe o consumo de cigarros, cigarrilhas, charutos, cachimbos ou de qualquer outro produto fumígeno, derivado ou não do tabaco, na forma que especifica, e cria ambientes de uso coletivo livres de tabaco. Diário Oficial do Estado do Rio de Janeiro 2009; 18 ago.

46. São Paulo. Assembléia Legislativa do Estado de São Paulo. Lei no 13.541, de 7 de maio de 2009. Proíbe o consumo de cigarros, cigarrilhas, charutos, cachimbos ou de qualquer outro produto fumígeno, derivado ou não do tabaco, na forma que especifica. Diário Oficial do Estado de São Paulo 2009; 8 mai. 
47. Agência Nacional de Vigilância Sanitária (Anvisa). A Anvisa na Redução à Exposição Involuntária à Fumaça do Tabaco. Brasília: Anvisa; 2009.

48. Organização Pan-Americana da Saúde (Opas). Ambientes 100\% Livres de Tabaco. Guia para Elaboração e Implantação de Políticas Públicas. $1^{\text {a }}$ edição. [página na Internet]. 2008 [acessado 2013 set 5]. Disponível em: http://new.paho.org/bra/index. php?option $=$ com_docman\&task $=$ doc_details\&gid $=$ 714\&Itemid $=423$

49. Issa JS, Abe TMO, Pereira AC, Megid MC, Shimabukuro CE, Valentin LSO, Ferreira MM, Nobre MR, Lancarotte I, Barretto AC. The effect of São Paulo's smoke-free legislation on carbon monoxide concentration in hospitality venues and their workers. Tob Control 2010; 20(2):156-162.

50. Brasil. Constituição da República Federativa do Brasil de 1988. Diário Oficial da União 1988; 5 out.

51. Brasil. Ministério da Saúde (MS). Secretaria de Atenção à Saúde. Instituto Nacional de Câncer (Inca). Ação global para o controle do tabaco. $1^{\circ}$ tratado internacional de Saúde Pública. 3a Edição. Rio de Janeiro: Inca; 2004.

52. Organização Pan-Americana da Saúde (Opas). São Paulo respira melhor: adoção de ambientes fechados livres do tabaco no maior estado brasileiro. Brasília: Opas; 2010. [página na Internet]. [acessado 2013 set 5]. 48 p.: il. - (Estudo de Caso). Disponível em: http://new.paho.org/bra/index.php?option $=\mathrm{com}_{-}$ docman\&task $=$ doc_details\&gid=972\&Itemid $=423$

53. Schmidt MI, Duncan BB, Azevedo e Silva G, Menezes AM, Monteiro CA, Barreto SM, Chor D, Menezes PR. Doenças crônicas não transmissíveis no Brasil: carga e desafios atuais. Lancet Série Brasil 2011: 61-74.

54. Wünsch Filho V, Moncau JE. Mortalidade por câncer no Brasil 1980-1995: Padrões regionais e tendências temporais. Rev Assoc Med Bras 2002; 48(3): 250-257.

55. Malta DC, Moura LM, Souza MFM, Curado MP, Alencar AP, Alencar GP. Tendência da mortalidade do câncer de pulmão, traquéia e brônquios no Brasil, 1980-2003. J Bras Pneumol 2007; 33(5):536-543.

56. Pinto M, Ugá MAD. Os custos de doenças tabacorelacionadas para o Sistema Único de Saúde. Cad Saude Publica 2010; 26(6):1234-1245.

57. Organização Mundial da Saúde (OMS). Noncommunicable diseases and mental health. Global status report on noncommunicable diseases 2010. Geneva: OMS; 2011.

58. Brasil. Ministério da Saúde (MS). Instituto Nacional de Câncer (Inca). Convenção-Quadro para o Controle do Tabaco. Rio de Janeiro: Inca; 2011.

59. Brasil. Ministério da Saúde (MS). Instituto Nacional do Câncer (Inca). Tabagismo e Saúde nos Países em Desenvolvimento. Documento organizado pela Comissão Européia em colaboração com a Organização Mundial de Saúde e o Banco Mundial para a Mesa Redonda de Alto Nível sobre Controle do Tabagismo e Políticas de Desenvolvimento. Rio de Janeiro: Inca; 2003.
60. Gigliotti A, Laranjeira R. Habits, attitudes and beliefs of smokers in four Brazilian capitals. Rev Bras Psiquiatr 2005; 27(1):37-44.

61. Townsend J. Price and consumption of tobacco. $\mathrm{Br}$ Med Bull 1996; 52 (1):132-142.

62. Tobacco Free Center. Taxation and Price Essential facts. [página na Internet] 2009 [acessado 2013 set 5]. Disponível em: http://www.tobaccofreecenter. org/files/pdfs/en/TAX_essential_facts_en.pdf

63. Wünsch Filho V, Mirra AP, López RVM, Antunes LF. Tabagismo e câncer no Brasil: evidências e perspectivas. Rev Bras Epidemiol 2010; 13(2):175-187.

64. Lopes ALM. Processo de educação em saúde na cessação do tabagismo: revisão sistemática e metassíntese [dissertação]. São Paulo: Universidade de São Paulo; 2008.

65. Glasgow RE. Worksite smoking cessation: current progress and future direction. Can J Pub Health 1987; 78(6):21-27.

66. Passos VMA, Giatti L, Barreto SM. Tabagismo passivo no Brasil: Resultados da Pesquisa Especial Do Tabagismo, 2008. Cien Saude Colet 2011; 16(9):36713678.

67. Fichtenberg CM, Glantz S. Effect of smoke-free workplaces on smoking behavior. Systematic review. Brit Med J 2002; 325(7357):188.

68. Goldfarb LMCS. Avaliação de um programa piloto de prevenção do tabagismo em quatro escolas do município do Rio de Janeiro [dissertação]. Rio de Janeiro: Fundação Oswaldo Cruz; 2000.

69. Salonen JT, Puska P, Mustaniemi H. Changes in morbidity and mortality during comprehensive community programm to control cardiovascular disease during 1972-77 in North Karelia. Br Med J 1979; 2(6199):1178-1183.

70. Fortmann SP, Taylor CB, Flora JA, Jatulis DE. Changes in adult cigarette smoking prevalence after 5 years of community health education: The Stanford Five-City Project. Am J Epidemiol 1993; 137(1):82-96.

71. The Commit Research Group. Community Intervention Trial for Smoking Cessation (COMMIT). I - Cohort results from a four-year community intervention. Am J Pub Health 1995; 85(2):183-192.

72. The Commit Research Group. Community Intervention Trial for Smoking Cessation (COMMIT). II - Commit Changes in adult Cigarette smoking prevalence. Am J Pub Health 1995; 85(2):193-200.

Artigo apresentado em 18/08/2012

Aprovado em 11/10/2012

Versão final apresentada em 28/10/2012 